\title{
How can smartphone applications improve sun protection behaviour?
}

\author{
Authors: Mislav Mokos ${ }^{1}$, Branka Marinović ${ }^{1,2}$ (mentor) \\ ${ }^{1}$ School of Medicine, University of Zagreb, Zagreb, Croatia \\ ${ }^{2}$ Department of Dermatology and Venereology, University Hospital Centre Zagreb, Zagreb, Croatia \\ DOI: https://doi.org/10.26800/LV-142-supp5-45

\section{Introduction:}

UV radiation is the most significant environmental risk factor for the development of non-melanoma skin cancers and melanoma. Smartphone applications have been developed to improve the current forms of campaigns for better sun protection behaviour. They are targeting young people in particular because they prefer modern ways of communication, such as mobile phones and electronic media, instead of traditional media like newspapers and TV, which they find out-dated.

Aim:

Our study aimed to assess the data about the effectiveness of sun protection smartphone applications.

\section{Materials \& Methods:}

We used PubMed, Web of Science, and Google Scholar to search the literature using the following keywords: "sun protection", "mobile applications", "smartphones".

\section{Results:}

The majority of applications are user-friendly and provide personalized sun protection advice. These recommendations are commonly based on the users' personal information (Fitzpatrick skin phototype, age, height, weight, use of photosensitizing medication), UV index, time and location. The personalized recommendations include sun-protection practices (minimum sun protection factor needed, time until reapplication of sunscreen, the form of physical photoprotection) and the preferable time for being indoors. However, there is a limited number of published studies evaluating the impact of the applications on the users' awareness. The results are incoherent. Some reported an increase in the use of sunscreen, while others reported either a decrease in the use of sunscreen but an increase in the search for shade or no change in the users' behaviour at all.

\section{Conclusion:}

Smartphone applications offering personalized information regarding sun protection have become widespread. Although the demand for such forms of campaigns is rising, further studies are needed to evaluate their effectiveness on sun protection behaviour.

Keywords: Mobile applications, Smartphones, Sun protection 